\title{
Transparent alumina ceramics: Effect of standard and plasma generated stabilizing approaches in colloidal processing
}

DRDLÍKOVÁ, K.; MACA, K.; SLÁMA, M.; DRDLÍK, D.

Journal of the American Ceramic Society

2019, vol. 102, iss. 12, pp. 7137-7144

ISSN (Online): 1551-2916

DOI: http://dx.doi.org/10.1111/jace.16630

Accepted manuscript will be available from 06/06/2020

Final version available online: https://ceramics.onlinelibrary.wiley.com/doi/full/10.1111/jace.16630 